\title{
Glucocorticoid receptor promotes the function of myeloid-derived suppressor cells by suppressing HIF1 $\alpha$-dependent glycolysis
}

\begin{abstract}
Yun Lu ${ }^{1,2,6}$, Huanrong Liu ${ }^{1,2,6}$, Yujing Bi ${ }^{3,6}$, Hui Yang ${ }^{1,6}$, Yan $\mathrm{Li}^{1,2}$, Jian Wang ${ }^{1,2}$, Zhengguo Zhang ${ }^{1,2}$, Yu Wang ${ }^{1,2}$, Chunxiao Li ${ }^{1,2}$, Anna Jia ${ }^{2}$, Linian $\mathrm{Han}^{2}$, Ying $\mathrm{Hu}^{2}$, Yong $\mathrm{Zhao}^{4}$, Ruoning Wang ${ }^{5}$ and Guangwei Liu ${ }^{1,2}$

Immunomodulatory signaling imposes tight regulations on metabolic programs within immune cells and consequentially determines immune response outcomes. Although the glucocorticoid receptor (GR) has been recently implicated in regulating the function of myeloid-derived suppressor cells (MDSCs), whether the dysregulation of GR in MDSCs is involved in immune-mediated hepatic diseases and how GR regulates the function of MDSCs in such a context remains unknown. Here, we revealed the dysregulation of GR expression in MDSCs during innate immunological hepatic injury (IMH) and found that GR regulates the function of MDSCs through modulating HIF $1 \alpha$-dependent glycolysis. Pharmacological modulation of GR by its agonist (dexamethasone, Dex) protects IMH mice against inflammatory injury. Mechanistically, GR signaling suppresses HIF1 $\alpha$ and HIF $1 \alpha-$ dependent glycolysis in MDSCs and thus promotes the immune suppressive activity of MDSCs. Our studies reveal a role of GR-HIF $1 \alpha$ in regulating the metabolism and function of MDSCs and further implicate MDSC GR signaling as a potential therapeutic target in hepatic diseases that are driven by innate immune cell-mediated systemic inflammation.
\end{abstract}

Cellular \& Molecular Immunology (2018) 15, 618-629; doi:10.1038/cmi.2017.5; published online 13 March 2017

Keywords: glucocorticoid-receptor; glycolysis; innate immunity; liver injuries; metabolism; myeloid-derived suppressive cells; tolerance

\section{INTRODUCTION}

Hepatic inflammation is one of the most prevalent pathologic responses in a variety of liver diseases. ${ }^{1}$ Immune-mediated hepatic injury (IMH) is central to the pathogenesis of inflammatory liver diseases, including autoimmune hepatitis and viral hepatitis. ${ }^{2}$ The acute inflammatory phenotype can be largely attributed to the front-line immune defense, generated by the innate immune system involving Kupffer cells, monocytes, neutrophils and eosinophils. ${ }^{1}$ Following an initial defensive response through recognizing pathogens and producing proinflammatory cytokines, the innate immune system also instructs long-lasting adaptive immunity and amplifies effector responses through a diverse range of mechanisms. ${ }^{3}$ As such, innate immune cell-mediated liver injury is driven by acute innate inflammation and is further evidenced by a sustained inflammatory damage imposed from the adaptive immune response within the inflamed liver. Mechanistically, the dynamic and complex interactions involving a diverse range of innate immune cells play an instrumental role in driving the pathological progression and therapeutic outcome in hepatic diseases that are driven by innate immune cell-mediated systemic inflammation. Understanding the molecular and cellular interactions behind these processes will not only elucidate the pathogenesis but also implicate new therapeutic targets of liver inflammatory disease.

\footnotetext{
${ }^{1}$ Department of Immunology, School of Basic Medical Sciences, Fudan University, Shanghai 200032, China; ${ }^{2}$ Key Laboratory of Cell Proliferation and Regulation Biology of Ministry of Education, Institute of Cell Biology, College of Life Sciences, Beijing Normal University, Beijing 100875, China; ${ }^{3}$ State Key laboratory of Pathogen and Biosecurity, Beijing Institute of Microbiology and Epidemiology, Beijing 100071, China; ${ }^{4}$ State Key Laboratory of Membrane Biology, Institute of Zoology, Chinese Academy of Sciences, Beijing 100101, China and ${ }^{5}$ Center for Childhood Cancer and Blood Diseases, Hematology/ Oncology and BMT, The Research Institute at Nationwide Children's Hospital, Ohio State University, Columbus, Ohio 43205, USA

${ }^{6}$ These authors contributed equally to this work.

Correspondence: Dr Y Zhao or Dr R Wang or Dr G Liu, Key Laboratory of Cell Proliferation and Regulation Biology of Ministry of Education, Institute of Cell Biology, College of Life Sciences, Beijing Normal University, Xinjiekouwaidajie 19, Beijinig 100875, China.

E-mail: zhaoy@ioz.ac.cn or ruoning.wang@nationwidechildren.org or liugw@bnu.edu.cn

Received: 11 October 2016; Revised: 19 December 2016; Accepted: 20 December 2016
} 
Myeloid-derived suppressor cells (MDSCs) are morphologically and functionally heterogeneous population of the myeloid-cell progenitors; they constitute a unique component of the immune system and function as negative regulators of the immune response. ${ }^{4}$ MDSCs are composed of monocytes, macrophages, granulocytes, dendritic cells (DCs) and immature myeloid cells at different stages of differentiation, and they

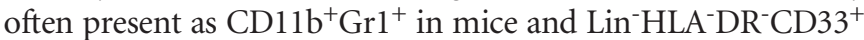

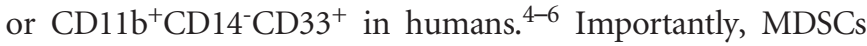
are able to expand and frequently stay in an activated state with increased production of nitrogen and reactive oxygen species in a diverse range of pathological inflammation, including cancer and some infectious or autoimmune disorders. ${ }^{7}$ Emerging evidence has shown that the development and accumulation of MDSCs in the tumor microenvironment play a critical role in fostering pro-tumoral immune modulation. ${ }^{4}$ While MDSCs have been most extensively studied in the context of tumors, recent studies also implicate their involvement in several other pathological contexts. ${ }^{8,9}$ However, the regulation and function of MDSCs in systemic inflammation-driven hepatic injury remains to be defined.

Synthetic glucocorticoid (GC) immunosuppressants, including dexamethasone (Dex), have been widely used in treating inflammatory disorders and are well known for their immunomodulatory effects. ${ }^{10}$ GCs exert their biological functions largely through regulating the glucocorticoid receptor (GR), which is a member of the nuclear receptor family and possesses transcription-regulatory function. ${ }^{11}$ Upon ligand binding, the GR dimerizes and translocates into the nucleus, where it can both directly and indirectly regulate the expression of a diverse range of inflammatory and anti-inflammatory genes. ${ }^{12}$ It is known that the tissue sensitivity to hormone signals is directly related to the levels of circulating cortisol and to the number of GRs found in cells. ${ }^{13}$ Previous studies have shown that the level of GR protein displays a dynamic change following the challenge of acute stressors and chronic stressors in various liver diseases. ${ }^{14}$

Our recent studies indicated that the GR signaling in MDSCs might play a critical role in the modulation of allograft immunity through reprogramming T-cell differentiation. ${ }^{15} \mathrm{In}$ light of this finding, we asked whether the dysregulation of GR in MDSCs is involved in innate immune cell-mediated liver diseases and how GR regulates the function of MDSCs. Here, we have revealed the dysregulation of GR expression in MDSCs during immunological hepatic injury (IMH) and found that GR regulates the function of MDSCs through modulating HIF1 $\alpha$-dependent glycolysis. Moreover, pharmacologically targeting GR signaling in MDSCs represents an effective therapeutic approach for systemic inflammation-driven hepatic injury.

\section{MATERIALS AND METHODS}

Mice

All animal experiments were approved by the Animal Ethics Committee of Fudan, Shanghai, China, and Beijing Normal University, Beijing, China. CD45.1+ C57BL/6 (B6) mice were obtained from the Center of Model Animal Research at Nanjing University (Nanjing, China). C57BL/6 $\left(\mathrm{CD} 45.2^{+}\right)$mice were received from Fudan University (Shanghai, China) or Beijing Normal University (Beijing, China) Experimental Animal Center. LysM Cre and HIF $1 \alpha^{\text {flox/flox }}$ mice on the C57BL/6 background were from The Jackson Laboratory and were further crossed to generate HIF $1 \alpha^{\text {flox/flox }}$, LysM Cre mice. All mice were bred and maintained in specific pathogen-free conditions. Sex-matched littermates at 6-8 wks of age were used in the experiments described in this study.

\section{LPS-induced hepatic injury and tolerance model}

As reported, ${ }^{16,17}$ for lipopolysaccharide (LPS) challenge, mice were injected (intraperitoneally (i.p.)) with LPS (5-10 mg/kg body weight). For endotoxin tolerance, mice were injected (i.p.) with LPS $(0.1 \mathrm{mg} / \mathrm{kg}$ body weight) daily for 4 consecutive days, and then they were re-challenged with LPS $(10 \mathrm{mg} / \mathrm{kg}$ body weight). Sera were collected 2 and $6 \mathrm{~h}$ after LPS challenge to test the levels of tumor necrosis factor- $\alpha$ (TNF $\alpha)$ and/or interleukin- $1 \beta$ (IL-1 $\beta$ ) production by flow cytometry (FCM) or ELISA. The levels of AST and ALT were examined by ELISA. The liver, kidney and lung were fixed with formaldehyde for histopathological examinations. Sections $(5 \mu \mathrm{m})$ were stained with hematoxylin and eosin for the assessment of cellular infiltration and inflammatory injuries.

\section{Flow cytometry}

For cell surface marker analysis, cells were stained with antibodies in PBS containing 0.1\% (wt/vol) BSA and 0.1\% $\mathrm{NaN}_{3}$. The following mAbs were used in the present study: anti-CD11b (M1/70; eBiosciences, Ben Lomond, CA, USA), anti-Gr1 (RB6-8C5; eBiosciences), anti-CD45 (TU116; BD Biosciences, San Diego, CA, USA), anti-GR (EPR4595; Abcam, Cambridge, MA, USA), anti-HIF1 $\alpha$ (IC1935P; R\&D system, Minneapolis, MN, USA), anti-human HLADR (L243; Biolegend, San Diego, CA, USA), anti-human CD33 (P67.0; Biolegend) and anti-human CD11b (ICRF44; Biolegend).

Intracellular staining was analysed by FCM as described. ${ }^{18}$ Cells isolated from different organs were re-stimulated with LPS (L2630; Sigma, St Louis, MO, USA) for CD $11 \mathrm{~b}^{+} \mathrm{Gr} 1^{+}$cell analysis for $5 \mathrm{~h}$, and GolgiStop (554724; BD Bioscience) was added for the last $2 \mathrm{~h}$. After surface staining and washing, the cells were immediately fixed with cytofix/cytoperm solution (554714; BD Biosciences) and were stained with anti-TNFo (MP6-XT22; eBiosciences) and anti-IL-10 (JES5-16E3; eBiosciences). For transcriptional factor analysis, after surface staining, the cells were fixed with fixation/permeabilization buffer (00-5523; eBioscience) and stained with anti-GR and anti-HIF1 $\alpha$. FCM data were acquired on a FACSCalibur (Becton Dickinson, San Diego, CA, USA), and data were analysed with FlowJo (TreeStar, San Carlos, CA, USA).

\section{Cell purification and culture}

Following cardiac perfusion with PBS, the liver or spleen was aseptically removed and mechanically disrupted between sterile frosted microscope slides as described before. ${ }^{19}$ Liver or splenic 


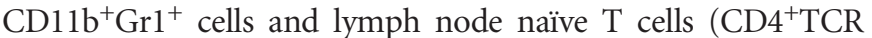
${ }^{+} \mathrm{CD} 25^{-} \mathrm{CD} 62 \mathrm{~L}^{\text {high }} \mathrm{CD} 44^{-}$cells) were sorted using a FACSAria II (Becton Dickinsion).

\section{Quantitative reverse transcriptase-PCR and immunoblot analysis}

Total RNA of MDSCs was extracted with the RNeasy kit (Qiagen, Valenica, CA, USA), and cDNA was synthesized using SuperScript III reverse transcriptase (Invitrogen, Carlsbad, CA, USA). An ABI 7900 real-time PCR system was used for quantitative PCR (qPCR), with primer and probe sets obtained from Applied Biosystems (Carlsbad, CA, USA). The results were analysed using SDS 2.1 software. The expression of each target gene is presented as the 'fold change' relative to that of control samples, as described previously. ${ }^{20}$ Immunoblot analysis was performed as described previously. ${ }^{21}$ Western blots were performed using specific antibodies: anti-GR (EPR4595; Abcam), anti-p-ErK (Thr202/Tyr204; Cell Signaling Technology, Danfoss, MA, USA), anti-p-JNK (Thr183/Tyr185; Cell Signaling Technology), anti-p-p38MAPK (Thr180/Tyr182; Cell Signaling Technology), anti- $\beta$-actin (AC-15; Sigma-Aldrich, St Louis, MO, USA) and anti-HIF1 $\alpha$ (IC1935P; R\&D system).

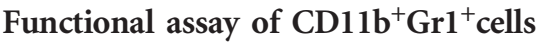

The depleting anti-Gr-1 mAb (RB6-8C5; $0.5 \mathrm{mg}$ ) was used to

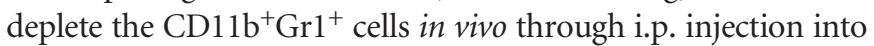
recipients at days -1 prior to the induction of hepatic injury.

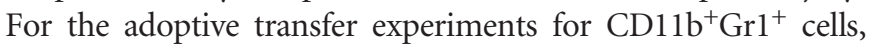
donor B6 $\left(\mathrm{CD} 45.2^{+}\right)$mice were challenged with LPS. Two days later, $\mathrm{CD} 11 \mathrm{~b}^{+} \mathrm{Gr}^{+}$cells were sorted from the liver of hepatic injury mice, and $\sim 1 \times 10^{6}$ cells were i.v. injected into syngeneic $\left(\mathrm{CD} 45.1^{+}\right)$B6 mice. One day after adoptive transfer, the recipient mice were challenged with LPS injection.

\section{Glycolytic flux analysis}

Glycolysis of MDSCs was determined by measuring the detritiation of $\left[3-{ }^{3} \mathrm{H}\right]$-glucose as reported previously. ${ }^{19}$ In brief, $1 \mathrm{uCi}\left[3-{ }^{3} \mathrm{H}\right]$-glucose (PerkinElmer) was added to the cell culture medium for $2 \mathrm{~h}$. The medium was collected and moved to microcentrifuge tubes containing $50 \mu \mathrm{l} 5 \mathrm{~N} \mathrm{HCL}$. The microcentrifuge tubes were then placed in $20-\mathrm{ml}$ scintillation vials containing $0.5 \mathrm{ml}$ water, and the vials were capped and sealed. ${ }^{3} \mathrm{H}-\mathrm{H}_{2} \mathrm{O}$ was separated from unmetabolized $\left[3-{ }^{3} \mathrm{H}\right]-$ glucose by evaporation for $24 \mathrm{~h}$ at room temperature.

\section{NO production assay}

After incubating equal volumes of culture supernatant or serum $(100 \mu \mathrm{l})$ with Greiss reagent, the absorbance at $550 \mathrm{~nm}$ was measured using a microplate reader (Bio-Rad, Hercules, CA, USA), as described previously. ${ }^{22}$

\section{GR receptor knockdown with shRNA}

Gene-specific short hairpin RNA (shRNA) sequences were cloned into the lentiviral shRNA expression plasmids (pMagic4.1; Sbo-bio, Shanghai, China) to construct the GR targeting shRNA and scramble control. Lentiviruses were produced and harvested from culture supernatant of $293 \mathrm{~T}$ cells (CL1032; Abgent, San Diego, CA, USA) transfected with shRNA vector. Sorted $\mathrm{CD}_{11 b^{+} \mathrm{Grl}}{ }^{+}$cells were infected with recombinant lentivirus, $\mathrm{GFP}^{+}$cells were sorted using a FACSAria II (Becton Dickinson), and the GR expression was confirmed using qPCR.

\section{Statistical analyses}

All data are presented as the mean \pm s.d. Student's unpaired $t$-test was applied for comparison of means to compare differences between groups. Comparison of the survival curves was performed using the Log-Rank (Mantel-Cox) test. A $P$-value (alpha-value) of $<0.05$ was considered to be statistically significant.

\section{RESULTS}

\section{MDSCs display reciprocal changes in GR expression in} hepatic injury or the tolerance IMH mouse model

Our recent studies implicated that GR signaling in MDSCs plays an important role in modulating allograft immunity. ${ }^{15}$ Here, we sought to extend these findings to evaluate the role of GR signaling and MDSCs in the context of systemic inflammation-driven hepatic injury. In this regard, we established a hepatic injury IMH model through inducing an acute and strong LPS response (LPS shock) ${ }^{23,24}$ and a tolerance IMH model through a consecutive induction of a moderate LPS response (LPS tolerance) ${ }^{21,22}$ (Figures 1a and $\mathrm{b}$ ). The alteration of serum ALT and AST levels in these two models (Figure 1c) confirms the status of hepatic injury and tolerance. Next, we sought to determine whether there are any alterations in MDSCs and to determine the expression of GR in MDSCs in

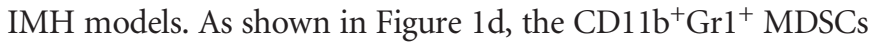
were significantly increased in the LPS-induced tolerance model, but not in the LPS-induced hepatic injury model, when compared with the control group. Consistent with the above findings, the protein and mRNA levels of GR in MDSCs that were isolated from the LPS-induced tolerant mice were higher than in the control, while they were lower in MDSCs that were isolated from the LPS-induced hepatic injury mice (Figure 1e and Supplementary Figure S1). Altogether, these data indicate that the reciprocal expression alteration of GR in MDSCs is regulated in the context of innate immune cell-mediated hepatic injury or tolerance.

\section{Dex enhances MDSC GR expression and protects mice against systemic inflammation-driven hepatic injury}

The significant downregulation of GR in MDSCs upon LPSshock led us to test the idea of enhancing GR expression as a potential approach for modulating MDSC function and pathological outcomes in IMH. To this end, we applied Dex, a potent agonist of GR, in the IMH model. In agreement with earlier findings, ${ }^{15}$ Dex treatment increased GR expression at

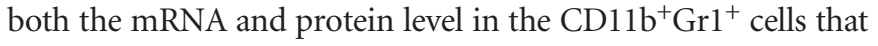
were isolated from mice following LPS shock (Figure 2a and Supplementary Figure S2). Strikingly, Dex treatment offered a remarkable protection against LPS-induced lethality in the IMH mouse model (Figure 2b). The histological analysis of 
a

Days

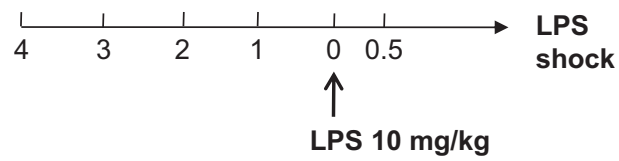

Days

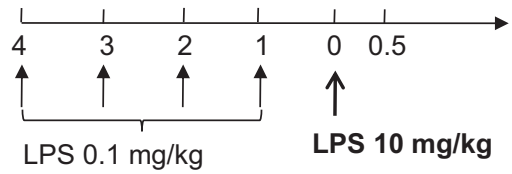

C

$\square$ PBS

- LPS shock

$\square$ LPS tolerance
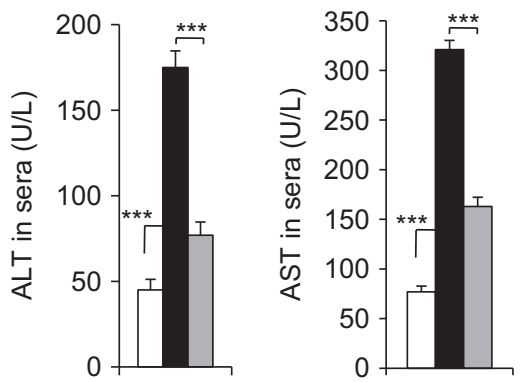

LPS

d

tolerance

b
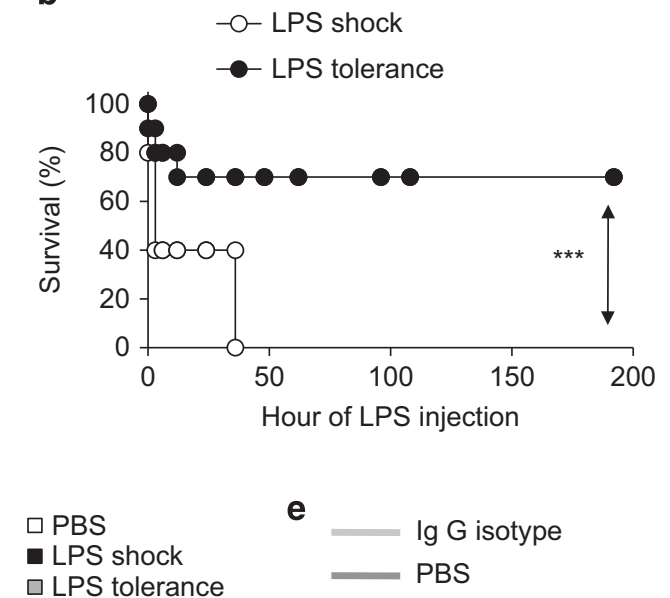

e

$\square$ LPS tolerance
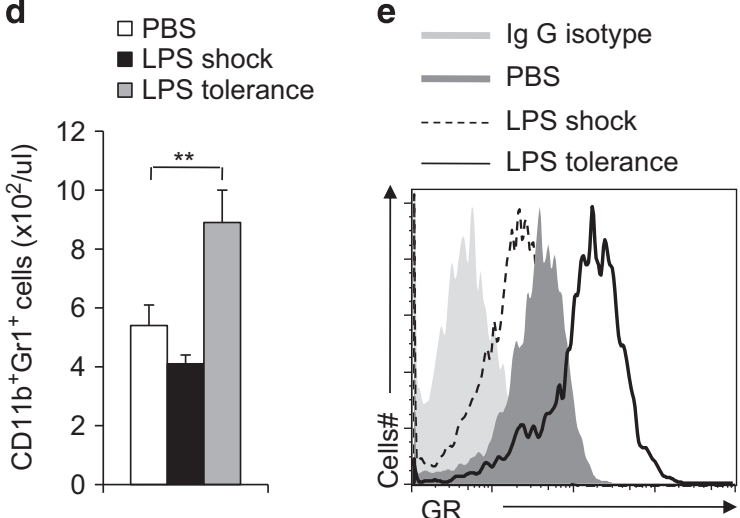

Figure 1 The alternation of myeloid-derived suppressor cell (MDSC) glucocorticoid-receptor in immune-mediated hepatic injuries. (a) diagram of the experimental model for LPS tolerance and for LPS shock induction; the mouse survival curve is plotted in $\mathbf{b}$. In the indicated murine models, the serum ALT and AST levels were determined with ELISA (c); the blood CD $11 b^{+} \mathrm{Gr} 1^{+}$cells were analysed by FACS (d); FACS analysis of GR expression in CD11 b ${ }^{+} \mathrm{Gr}^{+}$cells (e). Data are representative of three independent experiments $(n=4-10)$. ${ }^{* *} P<0.01$ and ${ }^{* *} P<0.001$ compared with the indicated groups. GR, glucocorticoid receptor; LPS, lipopolysaccharide.

liver, lung and kidney that were isolated from IMH mice and the analysis of serum levels of cytokines in IMH mice further confirmed that Dex treatment could ameliorate the LPSinduced immunological injuries and diminish the proinflammatory TNF $\alpha$ and IL-1 $\beta$ production in serum (Figures $2 \mathrm{c}$ and $d$ and Supplementary Figure S3A). To further determine the impact of Dex treatment on MDSCs, we isolated the $\mathrm{CD}_{1} 1 \mathrm{~b}^{+} \mathrm{Gr}^{+}$cells from experimental animals and examined their suppressive activities on T-cell proliferation and their intracellular cytokine production ex vivo. In addition to significantly potentiating the suppressive activities of MDSCs, Dex treatment reciprocally diminished and increased the proinflammatory TNF $\alpha$ expression and anti-inflammatory IL-10 expression in MDSCs (Figures 2e and $\mathrm{f}$ and Supplementary Figure S3B and C). Next, we asked whether MDSCs are responsible for mediating the protection against hepatic injuries in Dex-treated animals. To this end, we depleted MDSCs by injecting anti-Grl $\mathrm{mAb}^{25}$ and then followed the survival of mice upon LPS challenge. Efficient depletion of $\mathrm{CD} 11 \mathrm{~b}^{+} \mathrm{Grl}^{+}$ cells was confirmed by FACS (data not shown). Importantly, the depletion of $\mathrm{Gr}^{+}$cells abolished the protection offered by Dex treatment in IMH mice (Supplementary Figure S4). Conversely, the adoptive transfer of $\mathrm{CD}_{11} \mathrm{~b}^{+} \mathrm{Gr} 1^{+}$MDSCs that were isolated from Dex but not from PBS-treated IMH mice provided a significant protection against LPS-induced lethality in recipient mice (Figure $2 \mathrm{~g}$ ). Collectively, these data suggest that Dex treatment elevates the expression of GR in MDSCs and protects mice against immune-mediated hepatic injuries.

The elevation of GR expression in MDSCs is required for Dex-mediated protection against IMH

Having shown that Dex induces GR expression in MDSCs and protects mice against immune-mediated hepatic injuries, we next sought to determine the impacts of genetic modulation of GR on MDSC function. For this, we transuded either control shRNA or shRNA that targets GR into $\mathrm{CD} 11 \mathrm{~b}^{+} \mathrm{Gr} 1^{+}$cells that were isolated from the liver and then examined their cytokine production. The efficient knockdown of GR in MDSCs was confirmed by qPCR (Supplementary Figure S5A). Importantly, knockdown of GR enhanced the production of TNF $\alpha$, which is indicative of liver injury pathology and inflammation (Supplementary Figures S5B and C). Next, we sought to determine whether the inhibition of GR in MDSCs would counteract the effect of Dex treatment in protecting mice against immune-mediated hepatic injuries in the IMH model. 


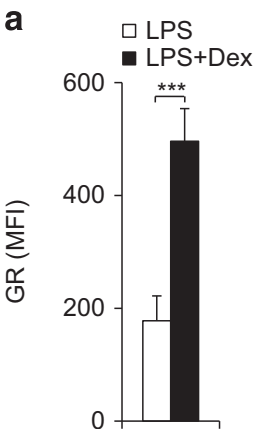

d

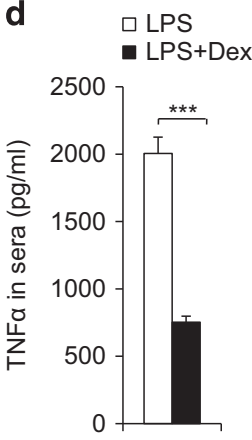

b

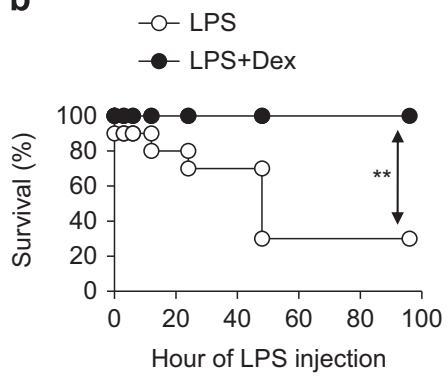

C

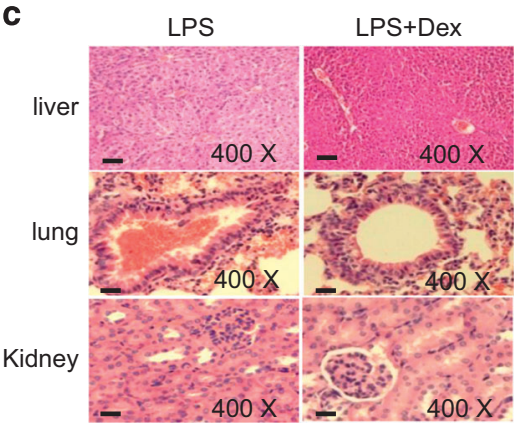

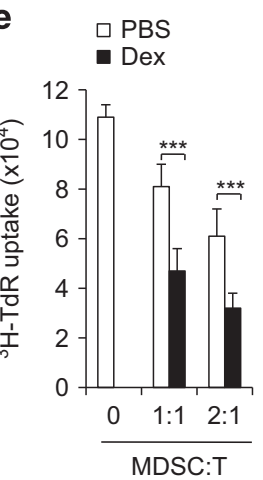

f

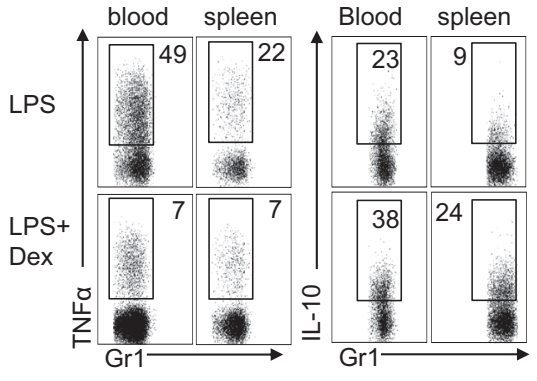

g

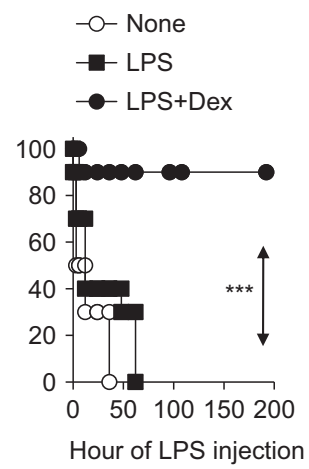

Figure 2 Dex treatment upregulates GR expression, potentiates MDSC activities and protects mice against immune-hepatic injuries. Agematched C57BL/6 mice were injected i.p. with PBS (solvent) or Dex ( $5 \mathrm{mg} / \mathrm{kg}$ body weight) daily starting at $6 \mathrm{~h}$ before LPS (5 mg/kg) injection. The GR expression on the CD1 $1 \mathrm{~b}^{+} \mathrm{Gr} 1^{+}$cells from liver $72 \mathrm{~h}$ following LPS injection was determined by FACS (a) and the survival curve of experimental mice is plotted $(\mathbf{b}, n=10)$. In a separate set of animals, the mice were sacrificed at $72 \mathrm{~h}$ following LPS-injection. The pathological changes in mouse liver, lung and kidney were examined by histochemistry (c). TNF $\alpha$ level in serum was determined with ELISA (d). The immunosuppressive activity of myeloid-derived CD1 $1 \mathrm{~b}^{+} \mathrm{Gr} 1^{+}$cells was determined by mixed lymphocyte reaction (e). The TNF $\alpha$ and IL-10 production in CD $11 \mathrm{~b}^{+} \mathrm{Gr} 1^{+}$cells in blood and spleen was analysed by FACS (f). Three groups of donor C57BL/6 mice were challenged with PBS (None), LPS or LPS plus Dex. After 3 days, a total of $1 \times 10^{6} \mathrm{CD} 11 \mathrm{~b}^{+} \mathrm{Gr} 1^{+}$cells were sorted from the liver of indicated mice and adoptively transferred into C57BL/6 recipient mice (10 mice per group) via i.v. Injection. After 10-12 h, all groups of recipient mice were challenged with LPS $(10 \mathrm{mg} / \mathrm{kg})$ and mouse survival was followed $(\mathbf{g})$. Data are representative of three (c-e) or four (f) independent experiments $(n=3-10) .{ }^{* *} P<0.01$ and ${ }^{* * *} P<0.001$ compared with the indicated groups. GR, glucocorticoid receptor; IL-10, interleukin-10; i.v., intravenous; LPS, lipopolysaccharide; MDSC, myeloid-derived suppressor cell; PBS, phosphate-buffered saline; TNF $\alpha$, tumor necrosis factor- $\alpha$.

For this, we chose a well-established antagonist of GR, RU$486,{ }^{26}$ and applied it to the IMH model, either alone or in combination with Dex. As expected, the RU-486 treatment alone efficiently reduced the GR expression in MDSCs that were isolated from LPS-challenged hepatic injury mice (Figure 3a). Similarly, RU-486 treatment alone significantly exacerbated the pathology of hepatic injury in IMH mice, as indicated by the levels of ALT, TNF $\alpha$ and IL- $1 \beta$ in sera (Figures $3 \mathrm{~b}$ and $\mathrm{c}$ ). This increased hepatic injury is likely due to its inhibition of MDSC-suppressive activity (Figure $3 \mathrm{~d}$ ). Importantly, the combination of RU-486 and Dex reversed the effect of Dex on GR expression in MDSCs, serum ALT and TNF $\alpha$ and IL- $1 \beta$ levels, and the suppressive activity of MDSCs (Figures 3a-d). To verify MDSCs as the cellular target of RU486 and Dex in the IMH model, we performed an adoptive-

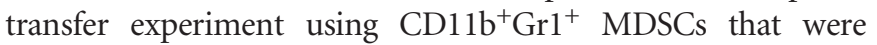
isolated from mice following LPS-challenge in combination with various treatments, as indicated in Figure 3e. While the adoptive transfer of MDSCs isolated from Dex-treated mice protected recipient mice against pathological lethality, the
RU-486 treatment of donor mice abolished such protection (Figure 3e). Together, these finding suggest that Dex or RU-486 treatment reciprocally modulates GR signaling and thus impacts the function of MDSCs during immune-mediated hepatic injury.

\section{The modulation of GR expression in MDSCs impacts LPS-induced immune tolerance}

Given the observation of increased GR expression in MDSCs in LPS-induced tolerance mode, we next determined the impact of modulating GR signaling in tolerance. While most of the experimental animals survived through LPS-challenge in the tolerant model, treatment with the GR antagonist RU-486 abolished the tolerance and restored the LPS-induced pathological lethality (Figure 4a). As expected, the RU-486 treatment significantly diminished GR expression in MDSCs isolated from experimental mice (Figure $4 \mathrm{~b}$ and Supplementary Figure S6) and diminished the suppressive activity of MDSCs (Figure 4c). Consistent with the results of the survival curve (Figure 4a), RU-486 treatment exacerbated the liver injury 


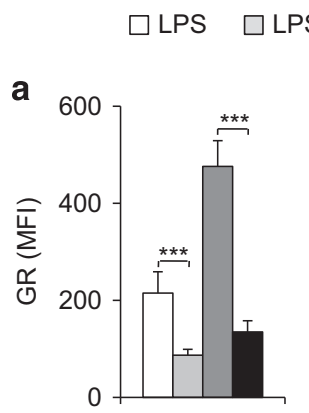

d

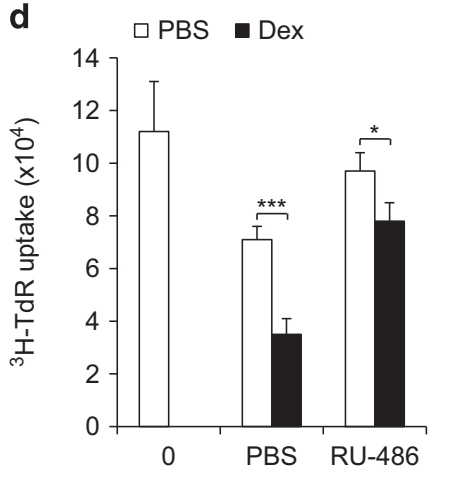

$\square$ LPS+Dex

LPS+Dex+RU-486
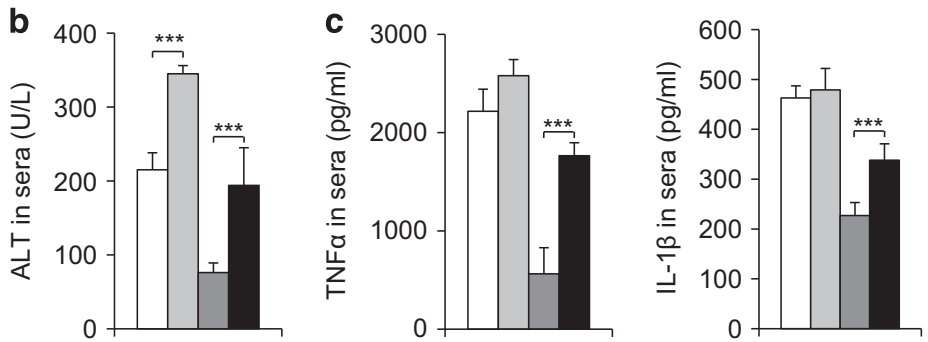

e

-O-PBS

$\triangle$ Dex

$\rightarrow$ Dex+RU-486

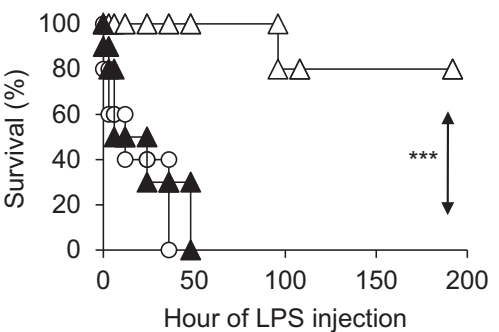

Figure 3 The downregulation of GR expression by RU-486 counteracts the effect of Dex in protecting mice against immune-hepatic injuries. As described in Figure 2, IMH was induced in four groups of age-matched C57BL/6 mice with the indicated treatments. Then, $72 \mathrm{~h}$ after LPS challenge, GR expression of CD11 b+Gr1 ${ }^{+}$MDSC in liver was analysed by FACS (a) and the level of ALT (b) TNF $\alpha$ and IL-1 $\beta$ (c) in serum were determined by ELISA. The immunosuppressive activity of myeloid-derived $\mathrm{CD} 11 \mathrm{~b}^{+} \mathrm{Gr} 1^{+}$cells was determined by mixed lymphocyte reaction (d) Three groups of donor C57BL/6 mice were challenged with LPS, LPS plus Dex or LPS plus Dex and RU-486 (10 mg/kg, i.p.). After 3 days, a total of $1 \times 10^{6} \mathrm{CD}_{11 \mathrm{~b}} \mathrm{Gr}^{+}$cells were sorted from the liver of indicated mice and adoptively transferred into C57BL/6 recipient mice (10 mice per group) via i.v. injection. After 10-12 h, all groups of recipient mice were challenged with LPS $(10 \mathrm{mg} / \mathrm{kg})$, and mouse survival was followed (e) Data are representative of three (a-c) or two (d) independent experiments (a-d; $n=3-5)$. $* * * P<0.001$ compared with the indicated groups. GR, glucocorticoid receptor; IMH, immunological hepatic injury; i.p., intraperitoneal; MDSC, myeloid-derived suppressor cell; LPS, lipopolysaccharide.

pathology and inflammation, as indicated by heightened serum ALT, TNF $\alpha$ and IL-1 $\beta$ levels (Figures $4 \mathrm{~d}$ and e). Taken together, these data suggest that the RU-486 treatment may break immune tolerance through targeting GR in MDSCs.

\section{Blocking glycolysis protects mice against IMH}

The metabolic shift toward aerobic glycolysis has been recently revealed as a general metabolic feature in myeloid cells upon inflammatory stimulation. ${ }^{27,28}$ Given that GR signaling has been implicated in the regulation of metabolism in several cellular contexts, ${ }^{29}$ we next asked how GR signaling impacts MDSC metabolism. For this, we examined the glycolytic activity of MDSCs following LPS-stimulation in the presence of Dex or RU-486. While the Dex treatment increased GR expression and suppressed glycolytic activity in MDSCs, the RU-486 treatment reduced GR expression and enhanced glycolytic activity (Figure 5a). Glucose utilization depends on a chain of reactions catalysed by multiple enzymes, eventually leading to the generation of lactate and to the net production of two ATP molecules as the energy source. The mRNA expression of a set of key glycolytic genes, such as glucose transport-1 (Glut1), gluocose-6-phosphate isomerase (GPI), enolase 1
(Eno1), lactate dehydrogenase- $\alpha(\mathrm{LDH} \alpha)$, and monocarboxylic acid transporter member 4 (MCT4), in the MDSCs was therefore determined by qPCR. As shown in Figure 5b, Dex or RU-486 treatment significantly reduced or enhanced the expression of Glut1, Eno1 and MCT4 in MDSCs, respectively. Consistent with the result of pharmacological modulation of GR, the genetic modulation of GR via shRNA knockdown significantly increased the glycolytic activity of MDSCs (Supplementary Figure S7).

Next, we sought to determine the impact of the glycolysis inhibitor, 2-deoxy-glucose (2-DG), on MDSCs in the IMH model. As expected, 2-DG treatment significantly blocked the glycolytic activity in MDSCs (Figure 5c). Importantly, 2-DG or Dex treatment displayed a comparable protection of mice against LPS-induced pathological lethality (Figure 5d). In addition, both 2-DG and Dex treatment enhanced the suppressive activity of MDSCs (Figure 5e) and ameliorated the liver injury pathology and inflammation, as indicated by reduced ALT and TNF $\alpha$ levels in the serum (Figures $5 \mathrm{f}$ and $\mathrm{g}$ ). Taken together, these data suggest that the glycolysis mechanisms might be related to the GR-mediated regulation of MDSCs in IMH. 

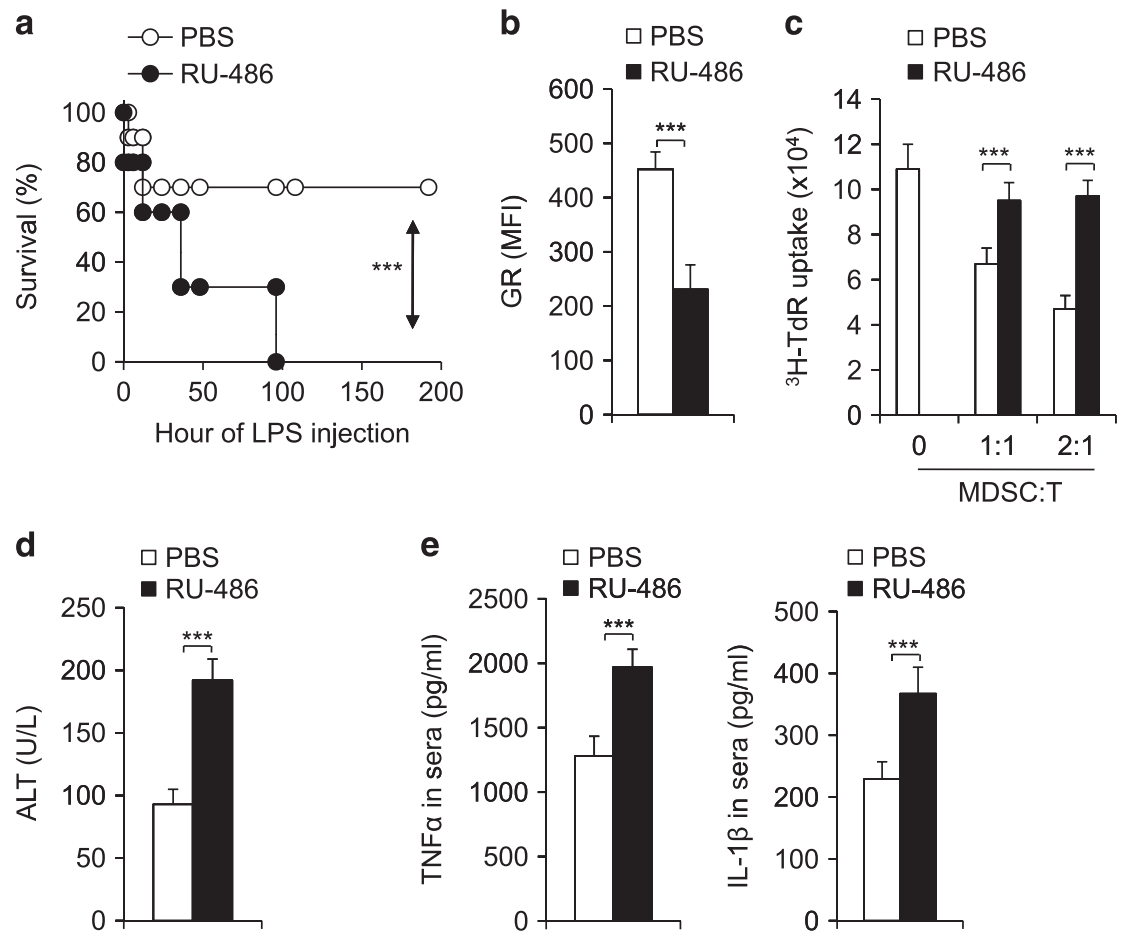

Figure 4 The downregulation of GR expression by RU-486 abolishes immune tolerance in an LPS-mediated tolerance model. Two groups of aged-matched C57BL/6 mice (10 per group) were pretreated with low dose LPS $(0.1 \mathrm{mg} / \mathrm{kg}$ ) daily from day 4 prior to challenge with LPS $(10 \mathrm{mg} / \mathrm{kg}$ ) for LPS tolerance induction. Either PBS or RU-486 was administrated at $6 \mathrm{~h}$ before the last LPS challenge. Mouse survival curve is plotted (a). Then, $72 \mathrm{~h}$ after the LPS challenge, GR expression of CD1 $1 b^{+} \mathrm{Gr} 1^{+}$MDSC in liver was analysed by FACS (b) the immunosuppressive activity of myeloid-derived $\mathrm{CD} 11 \mathrm{~b}^{+} \mathrm{Gr} 1^{+}$cells was determined by mixed lymphocyte reaction (c) and the levels of $A L T$ (d) TNF $\alpha$ and IL-1 $\beta$ (e) in serum were determined by ELISA. Data are representative of three (b-e) independent experiments $(n=3-5)$. $* * * P<0.001$ compared with the indicated groups. GR, glucocorticoid receptor; IL-1 $\beta$, interleukin-1 $\beta$; LPS, lipopolysaccharide; PBS, phosphate-buffered saline; TNF $\alpha$, tumor necrosis factor- $\alpha$.

\section{HIF1 $\alpha$ is the downstream target of GR in MDSCs}

The transcription factor hypoxia-inducible factor $1 \alpha$ (HIF $1 \alpha)$ has been implicated as a key regulator of metabolism in cancer cells, inflammatory $\mathrm{T}_{\mathrm{H}} 17$ cells and myeloid cells. ${ }^{21}$ We therefore examined the level of HIF1 $\alpha$ in MDSCs isolated from IMH mice treated with either Dex or RU-486. While the Dex treatment suppressed the expression of HIFl $\alpha$ (Figure 6a), it did not impact several other key inflammatory signaling pathways in MDSCs, as indicated by the comparable phosphorylation of JNK, ErK and p38MAPK between the two groups (Supplementary Figure S8). Conversely, both the pharmacological inhibition of GR by RU-486 and the genetic knockdown of GR by shRNA enhanced the expression of HIF1 $\alpha$ (Figure 6b and Supplementary Figure S9). Importantly, the deficiency of HIF1 $\alpha$ significantly ameliorated the liver injury pathology and the pro-inflammatory function of MDSCs and also enhanced MDSC function in the IMH model, as indicated by the reduced ALT serum level, the reduced intracellular TNF $\alpha$ in MDSCs and the enhanced suppression of T cell proliferation (Figures $6 \mathrm{c}-\mathrm{e}$ and Supplementary Figure $\mathrm{S} 10 \mathrm{~A}$ and B). In addition, the deficiency of HIF1 $\alpha$ led to a significantly prolonged survival in IMH mice (Figure 6f). While the RU-486 treatment exuberated the pathological phenotypes of
IMH, HIF1 $\alpha$ deficiency abolished the impact of RU-486 (Figures 6c-f).

Given that the Dex treatment suppresses the expression of HIF1 $\alpha$ (Figure 6a), we reasoned that HIF1 $\alpha$ deficiency would not result in any additive effect on the Dex treatment if HIF $1 \alpha$ functions as a key target effector of GR signaling. Supporting this idea, the Dex treatment of HIF1 $\alpha$-deficient mice displayed a similar degree of phenotype in liver injury pathology, in inflammatory cytokine production and in the suppressive activity of MDSCs compared to Dex treatment in WT mice (Figures 6g-i; Supplementary Figure S10C and D). Taken together, our results suggest that HIF1 $\alpha$ is required for GRdependent regulation of MDSCs in the IMH model.

\section{GR signaling controls MDSC nitric oxide production}

Nitric oxide $(\mathrm{NO})$ production plays a key role in mediating the immunosuppressive activity of MDSCs. We sought to determine whether the modulation of GR signaling impacts NO production in the IMH model. Consistent with the result showing that Dex treatment enhanced MDSC function (Figure 2e), Dex treatment increased the serum level of NO (Figure 7a). While Dex treatment increased the expression of inducible nitric oxide synthase (iNOS), an enzyme that produces NO, it moderately suppressed the expression 

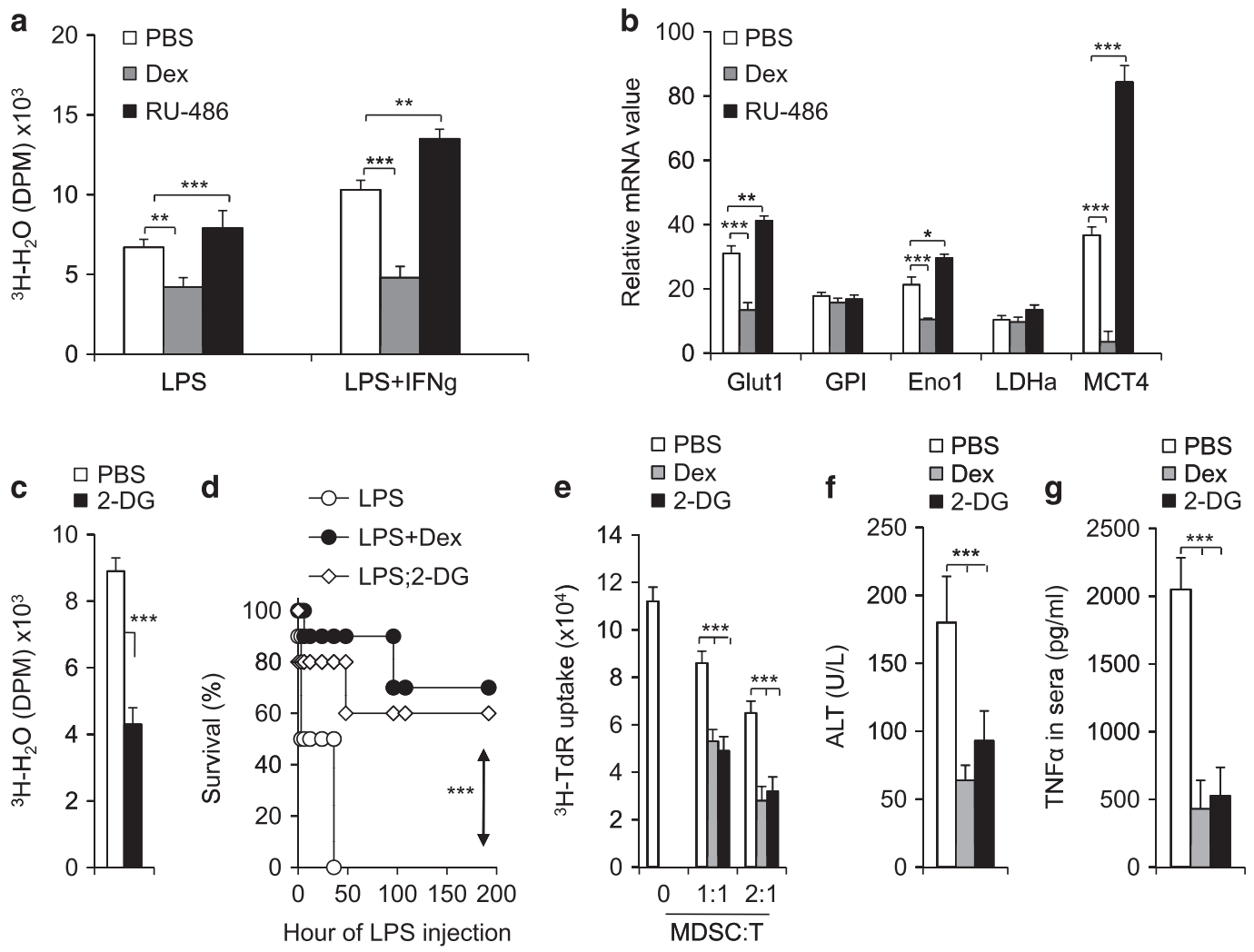

Figure 5 Blocking glycolysis protects mice against immune-hepatic injuries in IMH. As described in Figure 2, IMH was induced in the indicated three groups of age-matched C57BL/6 mice. Then, $12 \mathrm{~h}$ after LPS challenge, CD $11 \mathrm{~b}^{+} \mathrm{Gr} 1^{+}$cells were sorted from livers of indicated mice and were stimulated with LPS or LPS+IFN- $\gamma$ for $10 \mathrm{~h}$. The glycolytic activity (a) or the mRNA expression of indicated glycolytic genes (b) in the indicated groups was measured by the generation of ${ }^{3} \mathrm{H}$-labeled $\mathrm{H}_{2} \mathrm{O}$ from $\left[3-{ }^{3} \mathrm{H}\right]$-glucose or by qPCR. The liver MDSCs were isolated from IMH mice and were stimulated by LPS for $10 \mathrm{~h}$ in the presence of vehicle (PBS) or $1 \mathrm{mmol} / / 2$-DG. The glycolytic activity was measured as described above (c). As described in Figure 2, IMH was induced in three groups of age-matched C57BL 6 mice with the indicated treatments (LPS $10 \mathrm{mg} / \mathrm{kg}$ ). The survival curve is plotted (d) Then, $72 \mathrm{~h}$ after the LPS challenge, the immunosuppressive activity of myeloid-derived $\mathrm{CD} 11 \mathrm{~b}^{+} \mathrm{Gr} 1^{+}$cells was determined by mixed lymphocytes reaction (e) and the levels of ALT (f) and TNF $\alpha$ (g) in serum were determined by ELISA. Data are representative of two (a-c) or three (e-g) independent experiments $(n=3-10)$. ${ }^{*} P<0.05,{ }^{*} P<0.01$ and $* * * P<0.001$ compared with the indicated groups. IMH, immunological hepatic injury; IFN- $\gamma$, interferon- $\gamma$; LPS, lipopolysaccharide; MDSC, myeloid-derived suppressor cell; qPCR, quantitative PCR; TNF $\alpha$, tumor necrosis factor- $\alpha$.

Arginase I, a substrate-competing enzyme of iNOS, in MDSCs (Figures $7 \mathrm{~b}$ and c). Conversely, genetic knockdown of GR in MDSCs through shRNA resulted in a reduction of iNOS expression, indicating that the expression of iNOS is dependent on GR signaling (Supplementary Figure S11). Next, we applied L-NMMA, an inhibitor of iNOS, to MDSCs to determine whether blocking NO production would diminish the impact of Dex on the immune suppressive activity of MDSCs. As expected, the L-NMMA treatment abolished NO production (Supplementary Figure S12A). While the treatment of Dex significantly enhanced the immunosuppressive activity of MDSCs, the addition of L-NMMA abolished the effect of Dex on suppressing T-cell proliferation (Figure 7d). Finally, the L-NMMA treatment did not impact the glycolytic activity of MDSCs, excluding the possibility that NO indirectly altered HIF $1 \alpha$ and glycolysis in MDSCs (Supplementary Figure S12B). These data suggest that the GR-dependent NO production plays a role in regulating the immune suppressive activity of MDSCs in the IMH model.

\section{GR expression is dysregulated in MDSCs isolated from} human liver injury patients

By far, we have shown that the dysregulation of GR signaling in MDSCs may play a role in the pathogenesis of the murine IMH model and that the modulation of GR expression in MDSCs may represent an effective therapeutic strategy in treating murine IMH. Next, we sought to extend our finding in the murine IMH model to human patients with immune-mediated liver injuries. In this regard, we collected the peripheral blood from clinically confirmed hepatitis patients and determined the percentage of MDSCs and the expression of GR in MDSCs. As shown in Supplementary Figure S13A, the $\mathrm{HLADR}^{-} \mathrm{CD} 33^{+}$ $\mathrm{CD}_{11 \mathrm{~b}^{+}} \mathrm{MDSC}$ percentage and cell number are comparable in hepatitis patients and healthy controls. However, the intracellular staining of GR revealed that the protein level of 
a

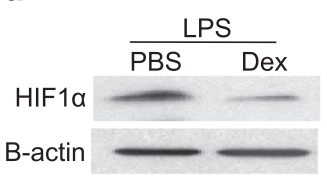

b

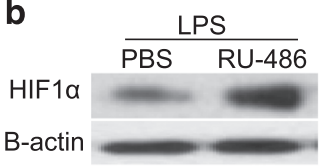

$\mathbf{f}$

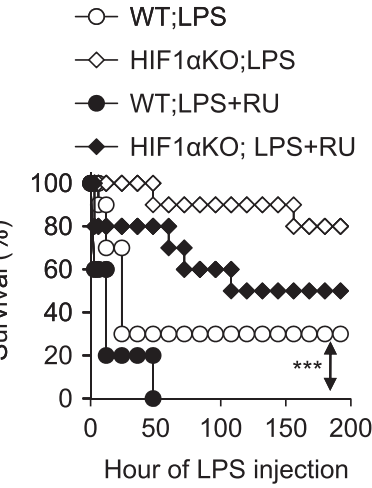

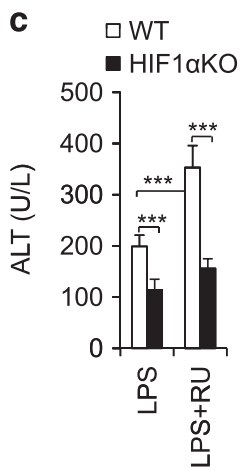

g

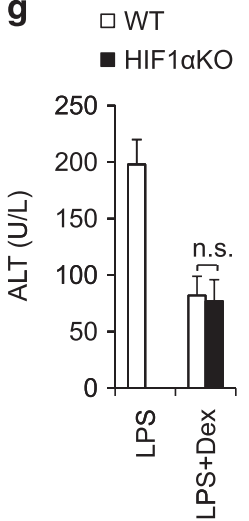

d

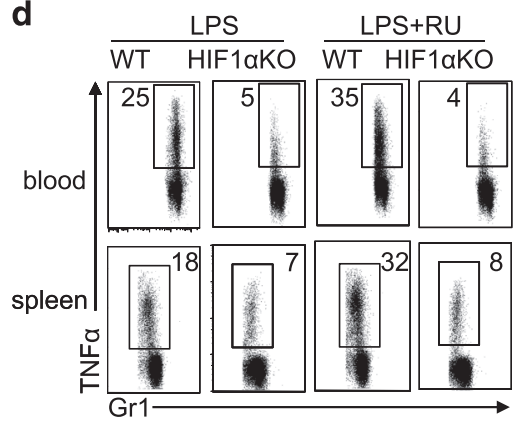

h
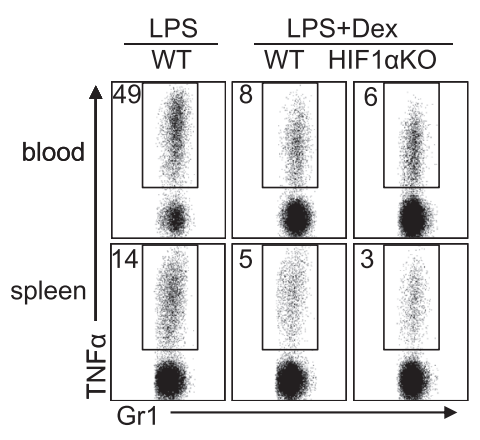

e

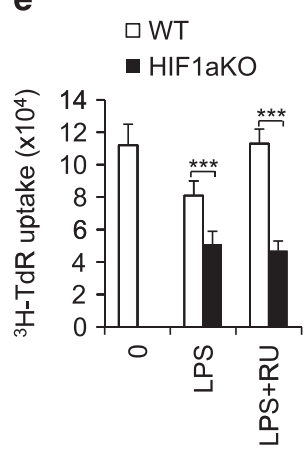

i
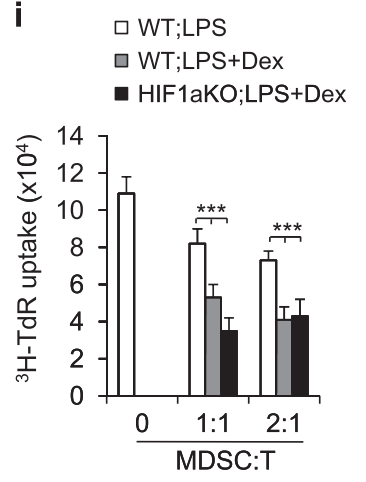

Figure $6 \mathrm{HIF} 1 \alpha$ is a downstream effector of GR signaling in MDSCs in IMH. The protein level of HIFl $\alpha$ in liver MDSCs that were isolated from IMH mice with the indicated treatments was determined by western blot (a, b). As described in Figure 2, IMH was induced in WT or $\mathrm{HIFl} \alpha^{\mathrm{fl} / \mathrm{fl}}$, LysM Cre ${ }^{+}$mice with the indicated treatments. Then, $72 \mathrm{~h}$ after LPS-injection, the ALT level in serum was determined with ELISA (c, g) the TNF $\alpha$ production in $\mathrm{CD} 11 \mathrm{~b}^{+} \mathrm{Gr} 1^{+}$cells in blood and spleen was analysed by FACS (d, h) and the immunosuppressive activity of myeloid-derived $\mathrm{CD} 11 \mathrm{~b}^{+} \mathrm{Gr} 1^{+}$cells was determined by mixed lymphocyte reaction (e, i) As described in Figure 2, IMH was induced in age-matched WT or HIFl $\alpha^{\text {fl/fl }}$, LysM Cre ${ }^{+}$mice $(n=10)$ with the indicated treatments (LPS $5 \mathrm{mg} / \mathrm{kg}$ ). The survival curve is plotted (f). Data are representative of two $(\mathbf{a}-\mathbf{e})$ or three $(\mathbf{g}-\mathbf{i})$ independent experiments $(n=3-5)$. ${ }^{* * *} P<0.001$ compared with the indicated groups. GR, glucocorticoid receptor; IMH, immunological hepatic injury; MDSC, myeloid-derived suppressor cell; LPS, lipopolysaccharide; NS, not significant; TNF $\alpha$, tumor necrosis factor- $\alpha$; WT, wild type.

GR is significantly lower in MDSCs isolated from hepatitis patients compared with MDSCs isolated from healthy controls (Supplementary Figure S13B). Consistent with the expression pattern of HIF $1 \alpha$ in MDSCs in the IMH mouse model, MDSCs that were isolated from hepatitis patients displayed an enhanced level of HIF $1 \alpha$ compared to MDSCs that were isolated from healthy controls (Supplementary Figure S13C). In summary, our results implicate a GR-HIF1 $\alpha$ axis in regulating MDSC metabolism and function. Our studies further suggest that the modulation of GR signaling in MDSCs may represent a novel and valid therapeutic strategy in treating immune-mediated liver injuries (Figure 7e). In addition, the dysregulation of GR signaling in MDSCs may also be present in human immune-mediated liver injury patients.

\section{DISCUSSION}

Synthetic glucocorticoids, such as prednisone, dexamethasone, and hydrocortisone, exert their immune modulatory effects in a broad range of immune diseases. ${ }^{10}$ Glucocorticoids elicit their biological action through the glucocorticoid receptor (GR), which belongs to a nuclear hormone receptor superfamily. In the absence of ligand binding, GR is in complex with chaperons and is retained in the cytosol. Upon glucocorticoid binding, GR is released from chaperon complexes and translocates to the nucleus, where it either directly transactivates targeted gene expression or indirectly modulates gene expression through interacting with other transcription factors. ${ }^{30}$

GC is also integrated into the complex immune feedbacksignaling network that plays a crucial role in fine-tuning immune signaling to avoid overactive immune responses. ${ }^{31}$ The therapeutic approach of targeting GR is therefore widely used in medicine to treat inflammation, infectious and autoimmune diseases. Previous studies have shown that the modulation of GR signaling impacts on many different types of immune cells in both innate and adaptive immunity. ${ }^{32}$ Recently, we and others have found that GR signaling may play a role in modulating the physiology and function of MDSCs. ${ }^{15,33-35}$ In the present study, we have revealed that MDSCs isolated from both systemic inflammation-driven hepatic injury, especially in innate immune cell-mediated hepatic injury mice and in human patients with hepatitis, display significantly lower levels of GR compared to healthy 
a

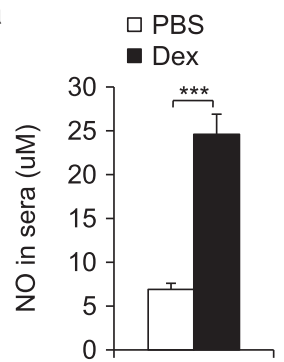

C

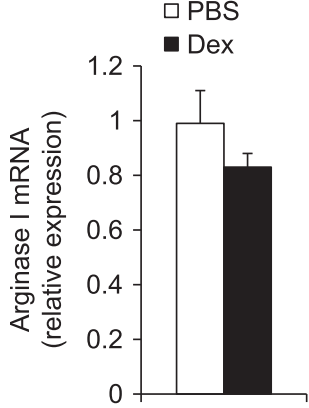

b

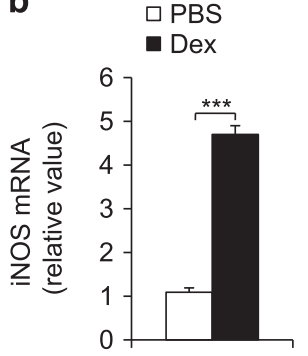

d

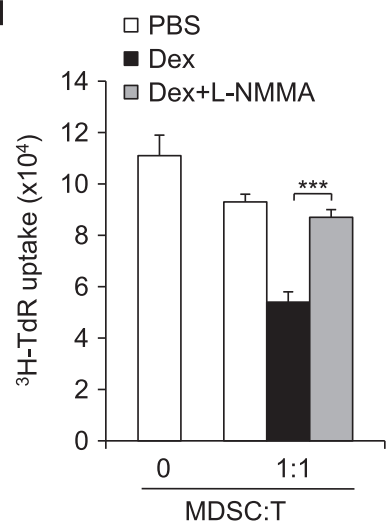

e

Inflammatory liver injuries

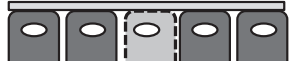

damage

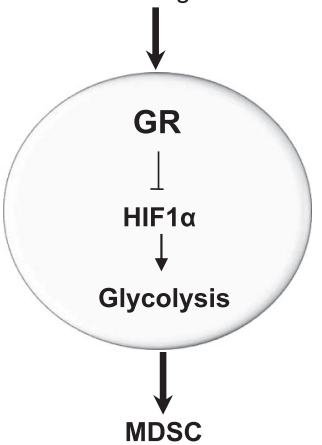

Suppressive activation

Immunological liver injury

Figure $7 \mathrm{GR}$ signaling modulates $\mathrm{NO}$ production in $\mathrm{CD} 11 \mathrm{~b}^{+} \mathrm{Gr} 1^{+}$MDSCs in IMH. As described in Figure 2, IMH was induced in mice with the indicated treatments. Then, $72 \mathrm{~h}$ after LPS-injection, the NO level in serum was determined by Greiss assay (a), the mRNA level of indicated genes in $\mathrm{CD}_{11} \mathrm{~b}^{+} \mathrm{Gr} 1^{+}$cells was determined by qPCR $(\mathbf{b}, \mathbf{c})$ and the immunosuppressive activity of myeloid-derived $\mathrm{CD} 11 \mathrm{~b}^{+} \mathrm{Gr} \mathrm{1}^{+}$ cells was determined by mixed lymphocyte reaction (d). Data are representative of three independent experiments $(n=4-5)$. ${ }^{* * *} P<0.001$ compared with the indicated groups. (e) A model summarizes the role of GR signaling in establishing MDSC functions through HIF1 $\alpha$ dependent glycolytic pathway. GR, glucocorticoid receptor; IMH, immunological hepatic injury; MDSC, myeloid-derived suppressor cell; qPCR, quantitative PCR.

controls. Treatment with the GR agonist dexamethasone (Dex) restored GR expression in MDSCs and ameliorated mortality and inflammatory insults in IMH, implying that GR signaling in MDSCs is a potential therapeutic target in treating innate immune cell-mediated hepatic injury.

Mechanistically, the upregulation of GR by Dex protects mice against immune-mediated hepatic injuries through potentiating MDSC suppressive activities, reducing their proinflammatory cytokines and increasing anti-inflammatory cytokine production. Conversely, the down-regulation of GR by RU-486 can significantly inhibit the immunosuppressive activities of MDSCs, increase pro-inflammatory cytokine production and reduce anti-inflammatory cytokine secretion, consequentially breaking immune tolerance in a chronically LPS-induced immune tolerance model. Previous studies suggested that ErK, ${ }^{36,37} \mathrm{JNK}$ and $\mathrm{p} 38 \mathrm{MAPK}$ signaling pathways are important downstream signaling nodes of GR in various cellular and pathological contexts. ${ }^{29,30}$ While we failed to reveal any significant changes in these signaling pathways, as indicated by a comparable level of p-ErK, p-p38, and p-JNK in MDSCs following the modulation of GR, we still cannot exclude the involvement of these pathways in fine-tuning GR signaling and impacting the function of MDSCs. Importantly, our current work implicates an important role of the GR-HIF1 $\alpha$ signaling axis in regulating the glycolytic activity of MDSCs. GR signaling suppresses HIF1 $\alpha$ and HIF1 $\alpha$-dependent glycolysis in MDSCs and thus promotes the immune suppressive activity of MDSCs in protecting against immunological hepatic injuries.

HIF $1 \alpha$ is a transcription factor known to play an important role in regulating the innate immune function in response to hypoxia. ${ }^{38}$ Furthermore, HIF1 $\alpha$ also responds to normoxia and plays a critical role in proinflammatory cytokine production. ${ }^{39,40}$ As a tumor grows, it rapidly outgrows its blood supply, leaving portions of the tumor with regions where the oxygen concentration is significantly lower than in healthy tissues. ${ }^{38}$ As such, hypoxia microenvironments in solid tumors stabilize HIF1 $\alpha$ and, in turn, alter the function of MDSCs through selectively up-regulating an immune checkpoint molecule, programmed death 1 ligand, and redirecting MDSC differentiation toward tumor-associated macrophages. ${ }^{41,42}$ Recent studies also implicate their involvement in several other pathological contexts, including inflammatory injuries. ${ }^{8,9}$ In the present study, we showed that HIF1 $\alpha$ is responsible for the GRregulated functions of MDSCs in systemic inflammation-driven immune-mediated hepatic injury mouse models. These data suggested that GR-HIF1 $\alpha$ signaling could be a potential therapeutic target in hepatic diseases that are driven by innate immune cell-mediated systemic inflammation.

To elicit rapid and robust immune responses under diverse metabolic and immune conditions, our immune system has evolved to coordinate nutrient metabolism and bioenergetic capacity with immune cell proliferation and activation. Such 
coordination is the result of the convergence of the signaling pathways that control metabolic activities and the signaling pathways that mediate immune functions. The inability to meet metabolic requirements would cause the imbalances of immune homeostasis. Heightened aerobic glycolysis has been implicated as a key metabolic feature in many proinflammatory immune cells, such as M1 macrophages, TLRstimulated dendritic cells, and $\mathrm{T}_{\mathrm{H}} 17 .{ }^{43-46} \mathrm{Myc}$ and HIF1 $\alpha$, two key transcriptional factors, play an important role in regulating metabolic gene expression that reprograms cellular metabolism to support tumor malignant growth. ${ }^{47,48}$ Notably, HIF1 $\alpha$ has also been implicated in regulating the glycolytic program in M1 macrophages, $\mathrm{T}_{\mathrm{H}} 9$ and $\mathrm{T}_{\mathrm{H}} 17$ cells and their pro-inflammatory functions. ${ }^{27,44,46,49}$ Consistent with the pro-inflammatory function of HIF1 $\alpha$ in macrophages and $\mathrm{T}_{\mathrm{H}} 17$ cells, we found that genetic ablation of HIF1 $\alpha$ or pharmacological inhibition of glycolysis suppresses the pro-inflammatory cytokine production in MDSCs while enhancing their anti-inflammatory activities. Similarly, the increased GR expression following Dex treatment not only leads to the downregulation of HIF $1 \alpha$ and glycolytic activity but also significantly enhances the anti-inflammatory function of MDSCs and protects mice against innate immune cell-mediated hepatic injury. In conclusion, our current work implicates a critical role of the GR-HIF1 $\alpha$ axis in regulating glycolysis and the function of MDSCs in innate immune cell-mediated hepatic injury. The pharmacological targeting of GR or MDSC metabolism may represent a promising therapeutic approach in treating human patients with systemic inflammation-driven hepatic injury, especially in innate immune cell-mediated hepatic injury.

\section{CONFLICT OF INTEREST}

The authors declare no conflict of interest.

\section{ACKNOWLEDGEMENTS}

This research is supported by grants from the National Natural Science Foundation for General Programs of China (31671524, 31171407 and 81273201, GL), the Key Basic Research Project of the Science and Technology Commission of Shanghai Municipality (12JC1400900, GL), the Innovation Program of Shanghai Municipal Education Commission (14ZZ009, GL), the Excellent Youth Foundation of Chinese Academy of Sciences (KSCX2-EW-Q-7, GL), R21AI117547, 1R01AI114581, V2014-001 from the V-foundation, and 128436RSG-15-180-01-LIB from the American Cancer Society (RW).

1 Bowen DG, Walker CM. Adaptive immune responses in acute and chronic hepatitis C virus infection. Nature 2005; 436: 946-952.

2 Das M, Sabio G, Jiang F, Rincon M, Flavell RA, Davis RJ. Induction of hepatitis by JNK-mediated expression of TNF-alpha. Cell 2009; 136: 249-260.

3 Liberal R, Grant CR, Longhi MS, Mieli-Vergani G, Vergani D. Diagnostic criteria of autoimmune hepatitis. Autoimmun Rev 2014; 13: 435-440.

4 Yang $\mathrm{H}, \mathrm{Bi} \mathrm{Y}$, Xue L, Wang J, Lu Y, Zhang $\mathrm{Z}$ et al. Multifaceted Modulation of SIRT1 in Cancer and Inflammation. Crit Rev Oncog 2015; 20: 49-64.
5 Zhao Y, Wu T, Shao S, Shi B. Phenotype, development, and biological function of myeloid-derived suppressor cells. Oncoimmunology 2016; 5: e1004983.

6 Lechner MG, Liebertz DJ, Epstein AL. Characterization of cytokineinduced myeloid-derived suppressor cells from normal human peripheral blood mononuclear cells. J Immunol 2010; 185: 2273-2284.

7 Pallett LJ, Gill US, Quaglia A, Sinclair LV, Jover-Cobos M, Schurich A et al. Metabolic regulation of hepatitis $B$ immunopathology by myeloidderived suppressor cells. Nat Med 2015; 21: 591-600.

8 Xia S, Sha H, Yang L, Ji Y, Ostrand-Rosenberg S, Qi L. Gr-1+ CD11b+ myeloid-derived suppressor cells suppress inflammation and promote insulin sensitivity in obesity. J Biol Chem 2011; 286: 23591-23599.

9 Arocena AR, Onofrio LI, Pellegrini AV, Carrera Silva AE, Paroli A, Cano RC et al. Myeloid-derived suppressor cells are key players in the resolution of inflammation during a model of acute infection. Eur $\mathrm{J}$ Immunol 2014; 44: 184-194.

10 van de Garde MD, Martinez FO, Melgert BN, Hylkema MN, Jonkers RE, Hamann J. Chronic exposure to glucocorticoids shapes gene expression and modulates innate and adaptive activation pathways in macrophages with distinct changes in leukocyte attraction. J Immunol 2014; 192: 1196-1208.

11 Rauch A, Seitz S, Baschant U, Schilling AF, Illing A, Stride B et al. Glucocorticoids suppress bone formation by attenuating osteoblast differentiation via the monomeric glucocorticoid receptor. Cell Metab 2010; 11: 517-531.

12 De Bosscher K, Van Craenenbroeck K, Meijer OC, Haegeman G. Selective transrepression versus transactivation mechanisms by glucocorticoid receptor modulators in stress and immune systems. Eur J Pharmacol 2008; 583: 290-302.

13 Yoo YM, Baek MG, Jung EM, Yang H, Choi KC, Yu FH et al. Parathyroid hormone-related protein and glucocorticoid receptor beta are regulated by cortisol in the kidney of male mice. Life Sci 2011; 89: 615-620.

14 Gong $\mathrm{H}$, Jarzynka MJ, Cole TJ, Lee JH, Wada T, Zhang B et al. Glucocorticoids antagonize estrogens by glucocorticoid receptormediated activation of estrogen sulfotransferase. Cancer Res 2008; 68: 7386-7393.

15 Liao J, Wang X, Bi Y, Shen B, Shao K, Yang H et al. Dexamethasone potentiates myeloid-derived suppressor cell function in prolonging allograft survival through nitric oxide. J Leukoc Biol 2014; 96: 675-684.

16 Bala S, Tang A, Catalano D, Petrasek J, Taha O, Kodys K et al. Induction of $\mathrm{Bcl}-3$ by acute binge alcohol results in toll-like receptor 4/LPS tolerance. J Leukoc Biol 2012; 92: 611-620.

17 Aneja RK, Tsung A, Sjodin H, Gefter JV, Delude RL, Billiar TR et al. Preconditioning with high mobility group box 1 (HMGB1) induces lipopolysaccharide (LPS) tolerance. J Leukoc Biol 2008; 84: 1326-1334.

$18 \mathrm{Li} \mathrm{C}, \mathrm{Bi}$ Y, Li Y, Yang H, Yu Q, Wang J et al. Dendritic cell MST1 inhibits Th17 differentiation. Nat Commun 2017; 8: 14275.

19 Schumann J, Prockl J, Kiemer AK, Vollmar AM, Bang R, Tiegs G. Silibinin protects mice from $\mathrm{T}$ cell-dependent liver injury. J Hepatol 2003; 39: 333-340.

20 Liu G, Burns S, Huang G, Boyd K, Proia RL, Flavell RA et al. The receptor S1P1 overrides regulatory T cell-mediated immune suppression through Akt-mTOR. Nat Immunol 2009; 10: 769-777.

21 Liu G, Bi Y, Shen B, Yang H, Zhang Y, Wang X et al. SIRT1 limits the function and fate of myeloid-derived suppressor cells in tumors by orchestrating HIF-1alpha-dependent glycolysis. Cancer Res 2014; 74: 727-737.

22 Wu T, Sun C, Chen Z, Zhen Y, Peng J, Qi Z et al. Smad3-deficient $\mathrm{CD11b}(+) \mathrm{Gr} 1(+)$ myeloid-derived suppressor cells prevent allograft rejection via the nitric oxide pathway. J Immunol 2012; 189: 4989-5000.

23 Murphey ED, Fang G, Varma TK, Sherwood ER. Improved bacterial clearance and decreased mortality can be induced by LPS tolerance and is not dependent upon IFN-gamma. Shock 2007; 27: 289-295.

24 Patenaude J, D'Elia M, Cote-Maurais G, Bernier J. LPS response and endotoxin tolerance in Flt-3L-induced bone marrow-derived dendritic cells. Cell Immunol 2011; 271: 184-191.

25 Liu G, Bi Y, Wang R, Yang H, Zhang Y, Wang X et al. Targeting S1P1 receptor protects against murine immunological hepatic injury through myeloid-derived suppressor cells. J Immunol 2014; 192: 3068-3079. 
26 Jang GR, Wrighton SA, Benet LZ. Identification of CYP3A4 as the principal enzyme catalyzing mifepristone (RU 486) oxidation in human liver microsomes. Biochem Pharmacol 1996; 52: 753-761.

27 Na YR, Gu GJ, Jung D, Kim YW, Na J, Woo JS et al. GM-CSF Induces Inflammatory Macrophages by Regulating Glycolysis and Lipid Metabolism. J Immunol 2016; 197: 4101-4109.

28 Bowden SD, Rowley G, Hinton JC, Thompson A. Glucose and glycolysis are required for the successful infection of macrophages and mice by Salmonella enterica serovar typhimurium. Infect Immun 2009; 77: 3117-3126.

29 Wang R, Green DR. Metabolic checkpoints in activated T cells. Nat Immunol 2012; 13: 907-915.

30 Kharwanlang B, Sharma R. Molecular interaction between the glucocorticoid receptor and MAPK signaling pathway: a novel link in modulating the anti-inflammatory role of glucocorticoids. Indian J Biochem Biophys 2011; 48: 236-242.

31 Radu CG, Cheng D, Nijagal A, Riedinger M, McLaughlin J, Yang LV et al. Normal immune development and glucocorticoid-induced thymocyte apoptosis in mice deficient for the T-cell death-associated gene 8 receptor. Mol Cell Biol 2006; 26: 668-677.

32 Tsutsui S, Vergote D, Shariat N, Warren K, Ferguson SS, Power C. Glucocorticoids regulate innate immunity in a model of multiple sclerosis: reciprocal interactions between the $A 1$ adenosine receptor and beta-arrestin-1 in monocytoid cells. FASEB J 2008; 22: 786-796.

33 Varga G, Ehrchen J, Tsianakas A, Tenbrock K, Rattenholl A, Seeliger S et al. Glucocorticoids induce an activated, anti-inflammatory monocyte subset in mice that resembles myeloid-derived suppressor cells. J Leukoc Biol 2008; 84: 644-650.

34 Diao W, Jin F, Wang B, Zhang CY, Chen J, Zen $\mathrm{K}$ et al. The protective role of myeloid-derived suppressor cells in concanavalin A-induced hepatic injury. Protein Cell 2014; 5: 714-724.

35 Semple JW. Move over Tregs, MDSCs are here. Blood 2016; 127: $1526-1528$

36 Wang HC, Zentner MD, Deng HT, Kim KJ, Wu R, Yang PC et al. Oxidative stress disrupts glucocorticoid hormone-dependent transcription of the amiloride-sensitive epithelial sodium channel alpha-subunit in lung epithelial cells through ERK-dependent and thioredoxinsensitive pathways. J Biol Chem 2000; 275: 8600-8609.

37 Lv WM, Zhao Y, Yang G, Dong SY, Zhang GH, Zhang Y et al. Role of Ras, ERK, and Akt in glucocorticoid-induced differentiation of embryonic rat somatotropes in vitro. Mol Cell Biochem 2014; 391: 67-75.

38 Denko NC. Hypoxia, HIF1 and glucose metabolism in the solid tumour. Nat Rev Cancer 2008; 8: 705-713.

39 Cramer T, Yamanishi Y, Clausen BE, Forster I, Pawlinski R, Mackman N et al. HIF-1alpha is essential for myeloid cell-mediated inflammation. Cell 2003; 112: 645-657.

40 Walmsley SR, Cadwallader KA, Chilvers ER. The role of HIF-1alpha in myeloid cell inflammation. Trends Immunol 2005; 26: 434-439.
41 Noman MZ, Desantis G, Janji B, Hasmim M, Karray S, Dessen P et al. PD-L1 is a novel direct target of HIF-1alpha, and its blockade under hypoxia enhanced MDSC-mediated T cell activation. J Exp Med 2014; 211: 781-790.

42 Corzo CA, Condamine T, Lu L, Cotter MJ, Youn JI, Cheng P et al. HIF-lalpha regulates function and differentiation of myeloid-derived suppressor cells in the tumor microenvironment. J Exp Med 2010; 207: 2439-2453.

43 Farooque A, Afrin F, Adhikari JS, Dwarakanath BS. Polarization of macrophages towards M1 phenotype by a combination of 2-deoxy-dglucose and radiation: Implications for tumor therapy. Immunobiology 2016; 221: 269-281.

44 Dang EV, Barbi J, Yang HY, Jinasena D, Yu H, Zheng Y et al. Control of $\mathrm{T}(\mathrm{H}) 17 / \mathrm{T}(\mathrm{reg})$ balance by hypoxia-inducible factor 1 . Cell 2011; 146: 772-784.

45 Pearce EJ, Everts B. Dendritic cell metabolism. Nat Rev Immunol 2015; 15: 18-29.

46 Hedl M, Yan J, Abraham C. IRF5 and IRF5 disease-risk variants increase glycolysis and human M1 macrophage polarization by regulating proximal signaling and Akt2 activation. Cell Rep 2016; 16: 2442-2455.

47 Xue G, Yan HL, Zhang Y, Hao LQ, Zhu XT, Mei Q et al. c-Myc-mediated repression of miR-15-16 in hypoxia is induced by increased HIF-2alpha and promotes tumor angiogenesis and metastasis by upregulating FGF2. Oncogene 2015; 34: 1393-1406.

48 Gordan JD, Thompson CB, Simon MC. HIF and c-Myc: sibling rivals for control of cancer cell metabolism and proliferation. Cancer Cell 2007; 12: 108-113.

49 Wang Y, Bi Y, Chen X, Li C, Li Y, Zhang Z et al. Histone deacetylase SIRT1 negatively regulates the differentiation of interleukin-9-producing CD4(+) T cells. Immunity 2016; 44: 1337-1349.

This work is licensed under a Creative Commons Attribution-NonCommercial-NoDerivs 4.0 International License. The images or other third party material in this article are included in the article's Creative Commons license, unless indicated otherwise in the credit line; if the material is not included under the Creative Commons license, users will need to obtain permission from the license holder to reproduce the material. To view a copy of this license, visit http://creativecommons.org/licenses/by-nc-nd/4.0/

C) The Author(s) 2018

Supplementary Information for this article can be found on the Cellular \& Molecular Immunology website (http://www.nature.com/cmi) 